\title{
Propofol versus propofol plus hydrocodone for flexible bronchoscopy: a randomised
} study

\author{
L. Schlatter, E. Pflimlin, B. Fehrke, A. Meyer, M. Tamm and D. Stolz
}

ABSTRACT: Propofol and the combination of a benzodiazepine and an opiate have been established for sedation in flexible bronchoscopy. It is as yet unknown whether propofol in combination with an opiate is superior to propofol alone to suppress cough during the procedure.

300 consecutive patients undergoing flexible bronchoscopy at a tertiary care university hospital were randomly allocated to receive either the combination propofol and hydrocodone or propofol alone in a double-blind fashion. The primary end-point was the cough score during the procedure as estimated by the physician using a visual analogue scale.

Demographics were similar in both groups. Compared with propofol alone, median (interquartile range) cough scores assessed by physicians, nurses and patients were significantly lower in the group randomised to the combination propofol and hydrocodone (2.5 (1.5-4.0) versus 2.0 (1.0-3.0), respectively, $p=0.011)$. Additionally, patients receiving the combination required significantly lower doses of propofol than those receiving propofol alone (200 mg (140-280) versus $260 \mathrm{mg}$ (180-350), $\mathbf{p}<\mathbf{0 . 0 0 0 1}$ ). Complex examinations, including bronchoalveolar lavage or transbronchial biopsy, benefited more from additional opiate. The duration of the procedure, time to discharge and complication rate were similar in both groups.

The combination of propofol and hydrocodone is safe and superior to propofol alone for cough suppression in flexible bronchoscopy.

KEYWORDS: Bronchoscopy, cough, intervention, safety, sedation

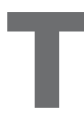
he current guidelines for bronchoscopy recommend offering sedation to all patients undergoing flexible bronchoscopy, except where there are contraindications [1]. The aim of sedation is to achieve good patient tolerance, comfort and cooperation while reducing complications of the procedure [2-4]. Sedation is associated with high patient satisfaction and willingness to return for a repeat bronchoscopy, if necessary [5]. A survey in the UK has shown that $>95 \%$ of the centres routinely perform sedated bronchoscopies [6].

Optimal sedation for flexible bronchoscopy has been assessed in a number of studies, which evaluated not only different sedative drug regimens but also particular drug requirements in specific sub-groups of patients [7-10]. In spite of the recommendations of the British Thoracic Society guidelines [1], combined sedation with opiate and a benzodiazepine has been shown to be effective and safe in a randomised, placebo-controlled trial, even in high-risk patients, such as those suffering from chronic obstructive pulmonary disease (COPD) [10]. Recently, propofol (2,6 diisopropylphenol) proved to be an attractive option to combined sedation with midazolam and hydrocodone, particularly if timely discharge is a priority [11-13]. Additionally, propofol seems to provide a higher quality of sedation in terms of neuropsychometric recovery and patient tolerance [14]. There is mounting evidence to suggest that this sedation technique can be safely performed by the nonanaesthesiologist $[11,14]$.

Propofol is a sedative hypnotic frequently used in the induction and maintenance of anaesthesia [15]. Its pharmacological mechanism is not entirely clear yet, however, it seems to activate the $\gamma$ aminobutyric A receptor-chloride ionophore complex [16]. Its rapid onset of action and amnesic properties, coupled with smooth and rapid recovery, make propofol an appealing agent for procedural sedation $[11,17,18]$. The significant

For editorial comments see page 507 .

AFFILIATIONS

Clinic of Pulmonary Medicine and Respiratory Cell Research, University Hospital Basel, Basel, Switzerland.

CORRESPONDENCE

D. Stolz

Clinic of Pulmonary Medicine and Respiratory Cell Research University Hospital Basel Petersgraben 4 4031 Basel Switzerland E-mail: dstolz@uhbs.ch

Received:

July 302010

Accepted after revision: Jan 182011

First published online:

Feb 102011 
advantage of a faster recovery time compared with other sedatives has been emphasised in several studies [19-22]. However, as yet, there are only limited data evaluating propofol for flexible bronchoscopy and it is as yet unknown whether the combination of propofol and an opiate offers any advantage over propofol alone for sedation during flexible bronchoscopy. Therefore, a prospective, randomised, placebo-controlled, double-blind intervention study was undertaken to determine whether the combination of propofol and hydrocodone is superior to propofol alone to suppress cough during the procedure. The primary end-point was the cough score during the procedure as estimated by the physician using a visual analogue scale (VAS).

\section{METHODS}

\section{Patients}

300 consecutive patients undergoing elective flexible bronchoscopy from October 2009 to December 2009 were randomly allocated to receive either intravenous propofol or the combination propofol and hydrocodone as a sedative agent (fig. 1). Intubated patients, those receiving an interventional bronchoscopy (e.g. laser or stent) and those with known allergy or intolerance to hydrocodone or propofol were not included in the study. Informed consent was obtained from each patient and the study was approved by the Institutional Review Board. The trial was registered with the Current Controlled Trials Database (ISRCTN81533083) (www.controlled-trials.com/isrctn).

\section{Study design}

All patients were assessed by a physician and a member of the nursing team trained in anaesthesiology prior to the procedure, including gradation of physical status in accordance with the American Society of Anesthesiologists (ASA) criteria. Bronchoscopies were performed transnasally or transorally with the patients in the semi-recumbent position by a total of five pulmonary fellows under the close supervision of four senior pulmonary physicians. Pulse oximetry was recorded continuously during the procedure and automated noninvasive blood pressure was monitored every $5 \mathrm{~min}$. Supplemental oxygen was offered at $4 \mathrm{~L} \cdot \mathrm{min}^{-1}$ via nasal cannula to all patients. In cases of desaturation $\leqslant 90 \%$, oxygen delivery was increased to $6 \mathrm{~L} \cdot \mathrm{min}^{-1}$ [23]. Nasal anaesthesia was achieved by applying $2 \%$ lidocaine gel nasally. Bronchoscopists were advised to instil 3-mL aliquots of $1 \%$ lidocaine over the vocal cords, onto the trachea and both right and left main bronchi. Supplemental local anaesthesia was given as judged by the bronchoscopist. All doses of instilled lidocaine doses were recorded for each patient. No inhaled lidocaine was given prior to the procedure [8]. Patients were given either $4 \mathrm{mg}$ i.v. hydrocodone or i.v. normal saline as placebo immediately prior to flexible bronchoscopy [10]. Randomisation was performed by arbitrary allocation to one of the two treatment groups based on a computer-generated random list (GraphPad Software, San Diego, CA, USA). Generator and executors of randomisation were separated. Bronchoscopists, nursing team members and patients did not have access to the randomisation code.

Loading doses of propofol were titrated to achieve adequate conscious sedation (onset of ptosis for bronchoscopy). Thereafter, conscious sedation was achieved with an i.v. infusion in an intermittent bolus technique. After an initial 10-20 mg i.v. propofol, the dose was then carefully titrated

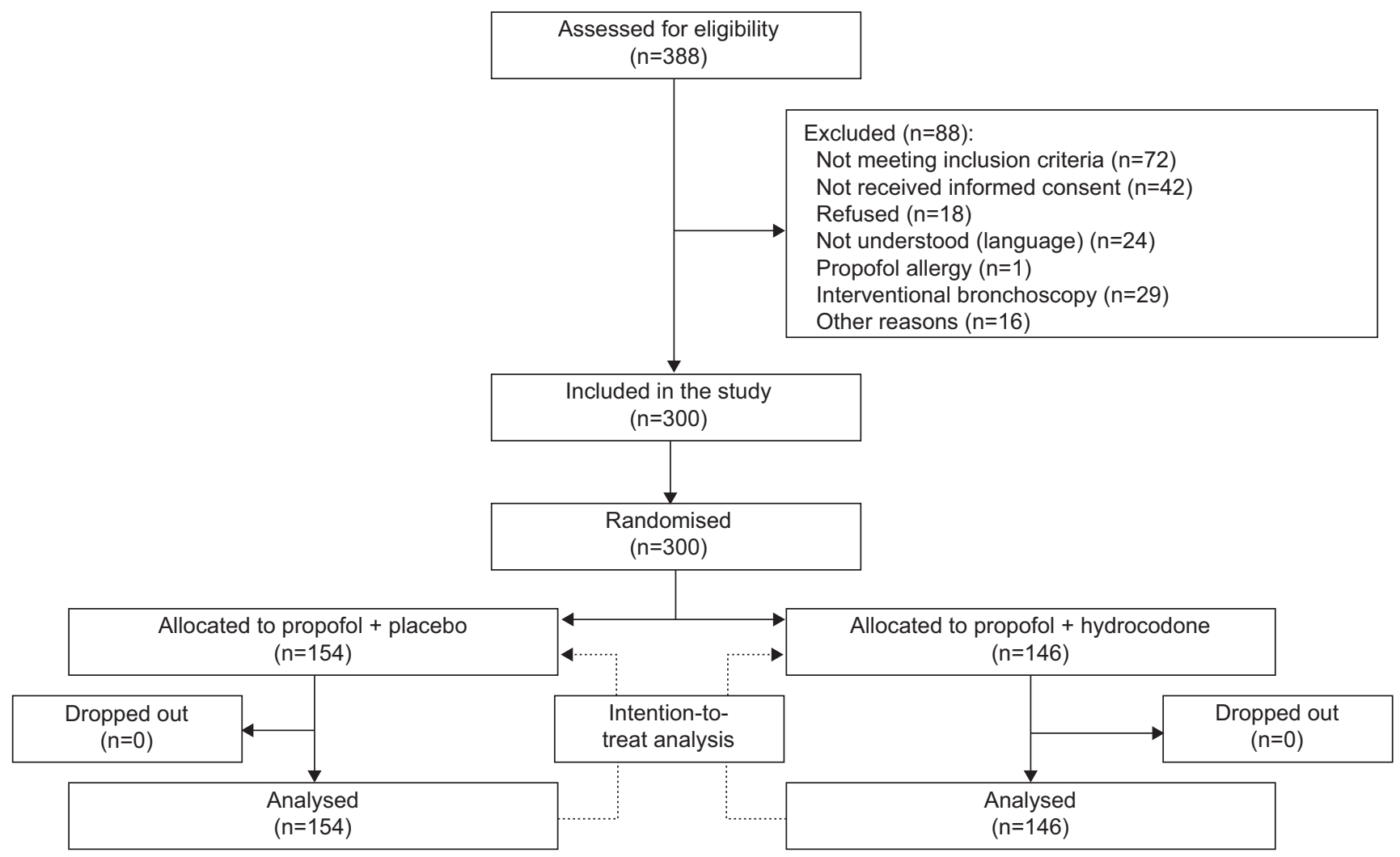

FIGURE 1. Study flow chart for patients included in the study. 
according to the ASA physical status classification: for ASA I and II, i.v. propofol boluses of $10-20 \mathrm{mg}$ i.v. were applied, whereas for ASA III and IV, precisely $10 \mathrm{mg}$ propofol i.v. was administered based on the clinical response, as previously described [24]. Between each bolus, a pause lasting $\geqslant 20 \mathrm{~s}$ had to be observed. If the effect disappeared during the examination, additional i.v. boluses of $10 \mathrm{mg}$ propofol were given, depending on the clinical effect, to maintain the required level of sedation. Signs of pain or discomfort, agitation, persistent cough and inadequate motor or verbal response to manipulation were considered indicators for insufficient sedation, leading to administration of an additional dose of propofol (10-20 mg). The total dose of propofol was documented for each patient.

Diagnostic procedures, e.g. brushing, washings, biopsy, bronchoalveolar lavage, endobronchial and transbronchial biopsies, were performed depending on the clinical indication. Haemodynamic parameters, sedation, duration of bronchoscopy, bronchoscopic procedures and complications were noted during the procedure in a standard form specifically designed for the study. Complications were defined as: oxygen desaturation $\leqslant 90 \%$, need for chin support, minor and major bleeding, need for artificial airway or invasive ventilation, need to abort bronchoscopy, need for intensive care unit (ICU)/intermediate care stay, and death.

At the end of the procedure, bronchoscopists and nursing staff charted their perception of cough during the procedure on a 10-cm VAS. Similarly, $2 \mathrm{~h}$ after bronchoscopy, patients were also asked to record their perception of cough related to the procedure on a $10-\mathrm{cm}$ VAS. In the scale, 0 denoted no cough and 10 represented incessant cough. Patients were also asked to record discomfort associated with the procedure on a $10 \mathrm{~cm}$ VAS. In the scale, 0 denoted no discomfort and 10 represented the greatest possible discomfort. Haemodynamic monitoring was performed immediately before, during and shortly after the procedure (after removal of the bronchoscope), and before transfer from the bronchoscopy suite to the recovery room. Moreover, patient's blood pressure and heart rate were continuously monitored for $\leqslant 2 \mathrm{~h}$ after bronchoscopy until discharge. Discharge readiness was evaluated starting at $2 \mathrm{~h}$ after the end of the procedure based on subjective (patient's opinion) and objective (orientation to time and place, capability to follow instructions, ability to drink water, ability to stand and walk autonomously, blood pressure, heart rate and oxygen saturation) criteria. In case any of the criteria being not fulfilled, subsequent evaluations were taken every 15-30 min. Additionally, patients were requested to state their perception of cough and of discomfort and to report any side-effects potentially related to the examination $24 \mathrm{~h}$ after the procedure.

\section{Data analysis}

Assuming a mean cough score of 4.35 in the arm treated with propofol alone and a score of 3.35 in the arm treated with combined sedation, each of them with a standard deviation of 1.5 [11], a total of 286 patients, 143 in each treatment arm, would be needed to achieve a significance level of $\mathrm{p}<0.05$ with a power of 0.9. Considering a $5 \%$ loss to follow-up, a total of 300 patients were predicted for inclusion. Differences in dichotomous variables were evaluated using the Chi-squared test or Fisher's exact test, as appropriate. Normally distributed parameters were analysed using the unpaired t-test for equality of means. All other continuously non-normally distributed parameters were evaluated using the non-parametric Mann-Whitney U-test or Kruskal-Wallis test, as appropriate. Correlation analyses between physicians and nursing staff VAS were performed using Spearman's rank correlation coefficient. To compare the effect of both sedation regimens in interventions of different complexity levels, patients were dichotomised into two groups: simple procedures (e.g. inspection or bronchial washings only) and complex procedures (e.g. bronchoalveolar lavage, bronchial brushing, endobronchial biopsy, transbronchial biopsy, transbronchial needle aspiration (TBNA) mediastinum or periphery, or endobronchial ultrasound (EBUS)).

The Statistical Package for Social Sciences programme was used (version 17 for Windows; SSPS Inc., Chicago, IL, USA). All tests were two-tailed; a p-value of $<0.05$ was considered significant. Results are expressed as mean \pm SD or median (interquartile range), unless otherwise stated.

\section{RESULTS}

Patient's demographics are presented in table 1 . There were no significant differences between both randomised groups in terms of age, presence of comorbidities or ASA physical status.

The indication and the diagnostic procedures in each randomised group are shown in table 2. The main indication for bronchoscopy was pulmonary infection, followed by suspicion of malignancy and bronchial toilette. Accordingly, the most common diagnostic procedures were bronchoalveolar lavage (49\%) and bronchial washing (34\%). TBNA, both from mediastinum and periphery of the lung, was performed in $18 \%$ of the cases. There were no significant differences between both randomised groups concerning indication for bronchoscopy and diagnostic procedures, with exception of the proportion of TBNA.

Compared with propofol alone, the group receiving the combination propofol and hydrocodone showed a significantly lower cough score as estimated by the physician $(p=0.011)$, nursing staff $(p=0.031)$ and the patient $2 \mathrm{~h}$ after the procedure $(p=0.025)$, as shown in table 3 . In addition, patient's discomfort score $2 \mathrm{~h}$ after bronchoscopy was also significantly lower in the group receiving the opiate. The Spearman's rank correlation coefficient of the cough scores was $0.598(p<0.0001)$ between physician and nurse, $0.165(p=0.008)$ between physician and patient and $0.273(\mathrm{p}<0.0001)$ between nurse and patient. After $24 \mathrm{~h}$, there were no differences in cough and discomfort scores between the groups.

Compared with propofol alone, the group receiving propofol and hydrocodone required significantly less propofol for sedation during the procedure (260 (180-350) mg versus 200 $(140-280) \mathrm{mg}, \mathrm{p}<0.0001)$. The median propofol doses were $3.5 \mathrm{mg} \cdot \mathrm{kg}^{-1}$ and $2.8 \mathrm{mg} \cdot \mathrm{kg}^{-1}$, respectively $(\mathrm{p}<0.0001)$. Lidocaine requirements were similar in both groups.

The duration of the examination did not differ significantly in both randomised groups. Nevertheless, there was a trend towards a shorter duration in the group of patients receiving the combination of propofol and hydrocodone (11 (7.8-17) $\mathrm{min})$ compared with propofol alone $(14(9-21) \mathrm{min}, \mathrm{p}=0.069)$. The time until discharge was similar in both study arms $(\mathrm{p}=0.249)$. 
TABLE 1 Baseline characteristics of 300 consecutive patients undergoing flexible bronchoscopy

\begin{tabular}{|c|c|c|c|}
\hline Characteristics & Propofol & Propofol + hydrocodone & $p$-value \\
\hline Age yrs & $61.8 \pm 16.3$ & $64.4 \pm 14.0$ & 0.127 \\
\hline Height $\mathrm{cm}$ & $170 \pm 10$ & $168 \pm 9$ & 0.063 \\
\hline Weight kg & $71.8 \pm 18$ & $69.4 \pm 14$ & 0.768 \\
\hline Pack-yrs & $23.8 \pm 28.8$ & $22.6 \pm 27.5$ & 0.933 \\
\hline Stroke & $0(0)$ & $0(0)$ & 1.000 \\
\hline Diabetes & $8(5)$ & $11(8)$ & 0.396 \\
\hline Immunosuppression & $28(18)$ & $22(15)$ & 0.487 \\
\hline Drugs & $4(3)$ & $0(0)$ & 0.051 \\
\hline Alcoholism & $5(3)$ & $6(4)$ & 0.682 \\
\hline COPD & $27(18)$ & $22(15)$ & 0.582 \\
\hline Class III & 101 & 87 & 0.065 \\
\hline Class IV & 0 & 1 & 0.488 \\
\hline
\end{tabular}

Data are presented as mean \pm SD or $n(\%)$, unless otherwise stated. COPD: chronic obstructive pulmonary disease; ASA: American Society of Anesthesiologists. ${ }^{*}$ : ASA class was not registered in 14 patients in the propofol group and 25 patients in the combined sedation group.

Haemodynamic findings before, during and after bronchoscopy are shown in table 4. Compared with propofol alone, patients assigned to the combination of propofol and hydrocodone did not show a lower blood pressure at any time during the procedure. The mean lowest oxygen saturation and the mean maximum oxygen requirement during the procedure were also similar across treatment groups.

TABLE 2 Indication for the examination and diagnostic procedures per randomisation group in 300 patients undergoing flexible bronchoscopy

\begin{tabular}{|c|c|c|c|}
\hline Indication for bronchoscopy & Propofol & Propofol + hydrocodone & p-value \\
\hline Patients $\mathbf{n}$ & 154 & 146 & \\
\hline Infection & $44(28)$ & $38(26)$ & 0.698 \\
\hline Suspicion of malignancy & $35(22)$ & $30(20)$ & 0.675 \\
\hline Bronchial toilette & $16(10)$ & $12(8)$ & 0.556 \\
\hline Pre- or post-interventional bronchoscopy & $10(6)$ & $16(11)$ & 0.218 \\
\hline Interstitial lung disease & $10(6)$ & $8(5)$ & 0.810 \\
\hline Cancer follow-up & $6(4)$ & $10(7)$ & 0.308 \\
\hline Mediastinal lymph nodes/mass & $7(4)$ & $6(4)$ & 1.000 \\
\hline Chronic cough & $2(1)$ & $7(4)$ & 0.096 \\
\hline Haemoptysis & $5(3)$ & $3(2)$ & 0.724 \\
\hline Miscellaneous & $19(12)$ & $16(11)$ & 0.723 \\
\hline \multicolumn{4}{|l|}{ Diagnostic procedures } \\
\hline Inspection only & $24(16)$ & $32(22)$ & 0.159 \\
\hline Bronchial washings & $55(35)$ & $46(31)$ & 0.441 \\
\hline Bronchoalveolar lavage & $77(50)$ & $70(47)$ & 0.722 \\
\hline Bronchial brushing & $20(12)$ & $16(10)$ & 0.589 \\
\hline Endobronchial biopsy & $27(17)$ & $23(15)$ & 0.679 \\
\hline Transbronchial biopsy & $23(14)$ & $16(10)$ & 0.306 \\
\hline TBNA mediastinum or periphery & $35(23)$ & $19(13)$ & 0.035 \\
\hline EBUS & $2(1)$ & $0(0)$ & 0.167 \\
\hline
\end{tabular}

Data are presented as $n(\%)$, unless otherwise stated. TBNA: transbronchial needle aspiration; EBUS: endobronchial ultrasound. 
TABLE 3 Outcome parameters in patients randomised to sedation with propofol or a combination of propofol and hydrocodone

\begin{tabular}{|c|c|c|c|}
\hline Characteristics & Propofol & Propofol + hydrocodone & p-value \\
\hline \multicolumn{4}{|l|}{ Cough score } \\
\hline Nurse VAS & $2.25(1.0-6.0)$ & $2.0(1.0-3.6)$ & 0.031 \\
\hline Patient $2 \mathrm{~h}$ after bronchoscopy VAS & $4.0(2.0-5.5)$ & $3.0(1.5-5.3)$ & 0.025 \\
\hline Discomfort score patient $2 \mathrm{~h}$ after bronchoscopy VAS & $0.5(0.0-2.0)$ & $0.5(0.0-1.5)$ & 0.037 \\
\hline Propofol dose mg & $260(180-350)$ & $200(140-280)$ & $<0.0001$ \\
\hline Propofol dose $\mathrm{mg} \cdot \mathrm{kg}^{-1}$ & $3.5(2.6-5.3)$ & $2.8(2.1-3.9)$ & $<0.0001$ \\
\hline Lidocaine dose mg & $90(60-120)$ & $90(60-120)$ & 0.584 \\
\hline Duration of the bronchoscopy min & $14(9-21)$ & $11(7.8-17)$ & 0.069 \\
\hline Time until discharge min & $120(99.5-141)$ & $128.5(109.5-145)$ & 0.249 \\
\hline
\end{tabular}

Data are presented as median (interquartile range), unless otherwise stated. VAS: visual analogue scale.

Table 5 shows the complication rate in both groups. The most common complications were oxygen desaturation $\leqslant 90 \%(32 \%)$ and the need for chin support (31\%). The number of patients presenting desaturation $\leqslant 90 \%$ on at least one occasion during the procedure was similar in both groups. The number of patients presenting major or minor bleeding, requiring chin support or termination of the procedure did not differ in both randomised groups. Only one patient needed to be transferred to the ICU. There were no deaths. These data are also similar to previous reports [11].

Outcomes in both randomised groups dichotomised by the complexity level of the examination are shown in tables 6 and 7 and figure 2. Patients undergoing simple procedures (e.g. inspection or bronchial washings only, $\mathrm{n}=100$ ) presented similar outcome parameters irrespectively of the applied sedation regimen. In contrast, patients undergoing complex procedures $(n=200)$ showed a significantly lower cough score, lower propofol requirements and a shorter duration of examination if receiving dual sedation with propofol and hydrocodone.
There was no difference in lidocaine requirement between both study arms in simple or complex examinations.

Due to the arbitrary imbalance in randomisation for TBNA and EBUS between both treatment groups, we also analysed outcomes of patients undergoing complex procedures excluding those in whom TBNA and/or EBUS were performed. In the subgroup of patients undergoing complex examinations without TNBA/EBUS $(n=150)$, the duration of the procedure was still shorter in the group receiving dual sedation compared with placebo (13 (9-18) min versus 14 (9-20.5) $\mathrm{min})$. However, this difference was not anymore statistically significant $(p=0.588)$. In contrast, propofol requirements and cough scores were still statistically significantly lower in the group of patients undergoing complex examinations and receiving hydrocodone in comparison with the group of patients receiving propofol alone $(200(160-270) \mathrm{mg}$ versus $260(200-340) \mathrm{mg}, \mathrm{p}<0.0001$ and 2 (1-3) versus 2.5 (1.5-4), $p=0.028)$, even if patients undergoing TBNA and/or EBUS were excluded from the analysis.

TABLE 4 Haemodynamic findings before, during and after bronchoscopy in patients randomised to sedation with propofol or a combination of propofol and hydrocodone

\begin{tabular}{|c|c|c|c|}
\hline Characteristics & Propofol & Propofol + hydrocodone & p-value \\
\hline Patients n & 154 & 146 & \\
\hline Initial systolic BP mmHg & $128 \pm 26$ & $126 \pm 28$ & 0.171 \\
\hline Initial diastolic BP mmHg & $78 \pm 21$ & $75 \pm 20$ & 0.113 \\
\hline Final systolic BP mmHg & $117 \pm 22$ & $116 \pm 24$ & 0.325 \\
\hline Final diastolic BP $\mathrm{mmHg}$ & $67 \pm 16$ & $68 \pm 16$ & 0.865 \\
\hline \multicolumn{4}{|l|}{$2 \mathrm{~h}$ re-evaluation } \\
\hline Systolic BP mmHg & $114 \pm 21$ & $118 \pm 22$ & 0.550 \\
\hline Diastolic BP mmHg & $65 \pm 15$ & $67 \pm 15$ & 0.446 \\
\hline Lowest oxygen saturation \% & $90.2 \pm 5.3$ & $90.4 \pm 5.2$ & 0.209 \\
\hline Oxygen requirement $L \cdot \mathrm{min}^{-1}$ & $5.4 \pm 2.8$ & $5.8 \pm 2.7$ & 0.262 \\
\hline
\end{tabular}

Data are presented as mean $\pm \mathrm{SD}$, unless otherwise stated. BP: blood pressure. 


\begin{tabular}{|c|c|c|c|}
\hline Characteristics & Propofol & Propofol + hydrocodone & p-value \\
\hline Oxygen desaturation $\leqslant 90 \%$ & $55(36)$ & $42(29)$ & 0.199 \\
\hline Chin support & $55(36)$ & $38(26)$ & 0.070 \\
\hline Minor bleeding & $13(8)$ & $8(5)$ & 0.315 \\
\hline Termination of the examination & $0(0)$ & $1(1)$ & 0.304 \\
\hline Intubation & $0(0)$ & $0(0)$ & 1.000 \\
\hline Intensive care unit & $0(0)$ & $1(1)$ & 0.304 \\
\hline Death & $0(0)$ & $0(0)$ & 1.000 \\
\hline
\end{tabular}

Data are presented as n (\%), unless otherwise stated.

\section{DISCUSSION}

This study has three major findings. First, the combination of propofol and hydrocodone for sedation provides significant benefits for cough suppression in flexible bronchoscopy compared with propofol alone. Second, the peri-interventional complication rate for both sedation regimens is similar. Finally, patients receiving the combination of propofol and hydrocodone required markedly less propofol for providing the same operational conditions than those receiving propofol alone, particularly in those procedures involving more complex examinations. Therefore, there is an additive benefit in combining a pure sedative with an antitussive agent for sedation in flexible bronchoscopy.

To our knowledge, this is the first randomised, double-blind, placebo-controlled study analysing the additional effect of an opiate to propofol for sedation in flexible bronchoscopy. The addition of hydrocodone to propofol lead to a significant coughsuppressing effect, translating into lower cough scores as reported by physicians, nurses and patients. In spite of that, patients' cough and discomfort no longer differed in the two randomised groups $24 \mathrm{~h}$ after bronchoscopy. We assume that this is a result of a generally low side-effect rate related to flexible bronchoscopy [25], particularly 1 day after the procedure. Accordingly, the VAS score for cough and discomfort $24 \mathrm{~h}$ after the procedure was low for all patients $(2.0(1.0-4.5)$ and $0.5(0-2.5)$, respectively).
The second major finding of this study concerns the complication rate of the procedure. Haemodynamic parameters and procedural complications were similar in both study arms, implying that sedation using the combination of propofol and hydrocodone is as safe as sedation with propofol alone. Indeed, there was a trend to less need for chin support in the patients receiving the combination. Therefore, even if there is concern about more complications, particularly hypoxia, when using combined sedation [1], our data suggest quite the opposite. According to our results, the combination of a sedative with an opiate is as safe as the use of a single sedative. In fact, although propofol sedation by a non-anaesthetist physician is not allowed in several countries, our findings also support the feasibility and safety of propofol sedation even when administered by nurses [26]. Major complications (major bleeding, termination of the examination, intubation, need for ICU or death) were uncommon in both groups. The percentage of $2 \%$ was similar to the one described by DANG et al. [27] (2.2\%) in patients sedated with a benzodiazepine and an opiate.

Besides the decrease in cough scores in the arm receiving propofol plus hydrocodone, we observed a reduction of the propofol doses needed to achieve an adequate degree of sedation using the combined sedation regimen. Taking into account the low cost of hydrocodone, which is the main reason

\begin{tabular}{|c|c|c|c|}
\hline Characteristics & Propofol & Propofol + hydrocodone & p-value \\
\hline \multicolumn{4}{|l|}{ Cough score } \\
\hline Physician VAS & $2.0(1.0-3.0)$ & $2.0(1.0-3.0)$ & 0.951 \\
\hline Nurse VAS & $1.5(0.5-2.0)$ & $1.25(1.0-3.0)$ & 0.843 \\
\hline Discomfort score patient $2 \mathrm{~h}$ after bronchoscopy VAS & $0.5(0.0-1.5)$ & $0.5(0.0-1.5)$ & 0.992 \\
\hline Propofol dose $\mathrm{mg}$ & $165(140-215)$ & $160(120-220)$ & 0.572 \\
\hline Propofol dose $\mathrm{mg} \cdot \mathrm{kg}^{-1}$ & $2.6(2.1-3.2)$ & $2.3(1.8-3.1)$ & 0.150 \\
\hline Lidocaine dose mg & $60(60-90)$ & $90(60-120)$ & 0.303 \\
\hline Duration of the bronchoscopy $\min$ & $9(5-12)$ & $10(6-15)$ & 0.391 \\
\hline
\end{tabular}




\begin{tabular}{|c|c|c|c|c|}
\hline TABLE 7 & \multicolumn{4}{|c|}{$\begin{array}{l}\text { Outcome parameters in the subgroup of patients in whom bronchoalveolar lavage, bronchial brushing, endobronchial } \\
\text { biopsy, transbronchial biopsy, transbronchial needle aspiration and/or endobronchial ultrasound have been performed } \\
\text { during flexible bronchoscopy }\end{array}$} \\
\hline \multicolumn{2}{|c|}{ Characteristics } & Propofol & Propofol + hydrocodone & p-value \\
\hline \multicolumn{2}{|l|}{ Patients n } & 110 & 90 & \\
\hline \multicolumn{5}{|c|}{ Cough score } \\
\hline \multicolumn{2}{|c|}{ Physician VAS } & $3.0(2.0-5.0)$ & $2.0(1.0-3.6)$ & 0.004 \\
\hline \multicolumn{2}{|c|}{ Patient $2 \mathrm{~h}$ after bronchoscopy VAS } & $4.5(2.5-6.3)$ & $3.0(1.5-5.5)$ & 0.036 \\
\hline \multicolumn{2}{|c|}{ Discomfort score patient $2 \mathrm{~h}$ after bronchoscopy VAS } & $0.5(0.0-2.0)$ & $0.5(0.0-1.1)$ & 0.016 \\
\hline \multicolumn{2}{|c|}{ Propofol dose $\mathrm{mg}$} & $300(220-380)$ & $220(168-290)$ & $<0.0001$ \\
\hline \multicolumn{2}{|c|}{ Propofol dose $\mathrm{mg} \cdot \mathrm{kg}^{-1}$} & $4.2(3.1-5.7)$ & $3.2(2.4-5.3)$ & $<0.0001$ \\
\hline \multicolumn{2}{|c|}{ Lidocaine dose $\mathrm{mg}$} & $90(60-120)$ & $90(60-120)$ & 0.292 \\
\hline \multicolumn{2}{|c|}{ Duration of the bronchoscopy $\mathrm{min}$} & $18(10-26)$ & $14(9.5-18)$ & 0.020 \\
\hline
\end{tabular}

to favour this compound over other opiates, the combination of propofol and hydrocodone results in net medication cost savings of $\sim 20$ Euros per bronchoscopy (corresponding to $60 \mathrm{mg}$ propofol). HoHL et al. [19] calculated similar cost savings of 17.33 USD per sedation for procedural sedation in the emergency department. Therefore, routine use of the combination regime would translate to medication savings of $>35,000$ Euros each year in large respiratory medicine centres, such as our institution, which performs around 2,000 examinations each year. Interestingly, lidocaine doses did not differ among the study arms, either in the overall study group or in the analysis dichotomised by examination complexity. One might hypothesise that bronchoscopists prefer achieving additional

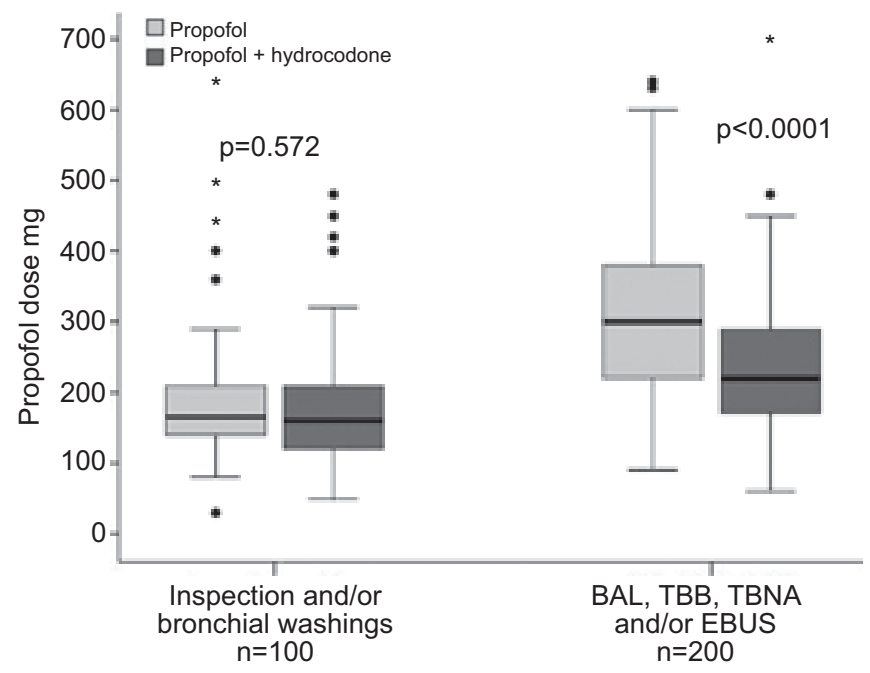

FIGURE 2. Propofol dose, dependent on the sedation regime (propofol versus propofol and hydrocodone) in the subgroup of patients undergoing simple bronchoscopic procedures, e.g. inspection and/or bronchial washings only, and in the subgroup of patients undergoing complex bronchoscopy procedures, e.g. bronchoalveolar lavage (BAL), transbronchial biopsy (TBB), transbronchial needle aspiration (TBNA) and/or endobronchial ultrasound (EBUS). Boxes represent median and interquartile range; whiskers represent range. @ : outliers; *: extreme outliers. sedation and cough suppression by further titrating propofol instead of applying additional lidocaine instillations.

The comparison of outcomes in procedures of different complexity levels revealed a pronounced benefit from the combination of propofol and hydrocodone in patients undergoing complex examinations. In cases in which only inspection or bronchial washings were performed, results from both sedation regimens were similar. In contrast, when complex bronchoscopies were performed, the combination of propofol and hydrocodone resulted in reduced cough and discomfort scores, required propofol doses and, potentially, duration of the procedure. This time-saving effect has been seen as a trend in the overall group and could be more apparent in complex procedures. This is particularly interesting in view of the low cough scores reported in both treatment arms, as one could hypothesise that the reduction in procedure time could be caused by the coughing suppressing effect of hydrocodone. In combination with the fact that propofol allows earlier discharge compared with a regimen including midazolam and hydrocodone [11], the potential additional time-saving during the examination might represent a further argument to support sedation with propofol and hydrocodone, particularly in complex examinations. Similar findings have been recently reported for endoscopic procedures of the gastrointestinal tract. A meta-analysis comparing sedation in gastrointestinal endoscopic procedures found a trend towards a reduction of complications, higher levels of satisfaction and cost-effectiveness for the use of propofol during colonoscopies, and higher levels of patient satisfaction and improved efficacy using propofol during upper gastrointestinal endoscopies, compared with a benzodiazepine-based sedation regime [28].

This study has a few limitations. First, our data is based on well-trained and experienced nursing staff with extensive knowledge in sedation with propofol for all endoscopic procedures, including upper and lower intestinal tract endoscopies. Therefore, our conclusions, particularly on safety, should be put into the local nursing staffs' perspective and should consider the aspects of the pertaining institution, e.g. volume of procedures. Second, there was a slightly higher proportion of TBNA in the group receiving propofol alone. 
However, it is well known that such imbalances are inherent to the randomisation process and that five out of 100 characteristics of both groups will differ just by chance. Thus, in a study controlling for 33 variables such as ours, a significant difference between the two study arms would be expected statistically for two variables. Moreover, we still observed statistically significant differences in cough scores and propofol requirements in complex examinations between both randomised arms, even after excluding patients undergoing TBNA and/or EBUS from the analysis. Because it is plausible to assume that patients undergoing particularly lengthy examinations, such as TBNA and EBUS, could benefit from additional cough suppression with hydrocodone, we believe that a further study including a larger number of TBNA/EBUS cases would be necessary to definitively confirm whether dual sedation with propofol and hydrocodone is able not only to reduce cough and propofol requirements but also to significantly shorten the duration of the procedure. Strengths of this study are the large number of patients included, the randomised, double-blind and placebocontrolled design, and the diversity of patient comorbidities (i.e. COPD) and bronchoscopic procedures.

In conclusion, our data suggest that the combination of propofol and hydrocodone is safe and that its cough-suppressing effect is superior to propofol alone for sedation in patients undergoing flexible bronchoscopy. The significant reduction in propofol requirements when the combination is used leads to an additional cost-saving effect. The higher the complexity of the procedures, the more pronounced are the benefits of the combination, which include a potential shortening of the duration of the procedure. Hence, we suggest, as long as there are no contraindications, the combination of propofol and hydrocodone be adopted as a standard sedation regimen, particularly in patients undergoing complex interventions in flexible bronchoscopy.

\section{SUPPORT STATEMENT}

This work was founded by the Clinic of Pulmonary Medicine and Respiratory Cell Research, University Hospital Basel, Basel, Switzerland. D. Stolz was supported by a grant from the Swiss National Foundation (PP00P3_128412/1).

\section{CLINICAL TRIAL}

This trial was registered with the Current Controlled Trials Database (ISRCTN81533083).

\section{STATEMENT OF INTEREST}

None declared.

\section{ACKNOWLEDGEMENTS}

We wish to thank the endoscopy staff (E. Baumann, M. Brenneisen, B. Fehrke, T. Galluccio, S. Groelly, E. Gysin, S. Hartig, B. Koch, B. Lehner, M. Ortmann, E. Pflimlin and J. Vögtli; all from Clinic of Pulmonary Medicine and Respiratory Cell Research, University Hospital Basel, Basel, Switzerland) for their excellent collaboration. We thank A. Schötzau (Schötzau and Simmen, Statistical Advice, Basel, Switzerland) for statistical advice.

\section{REFERENCES}

1 British Thoracic Society Bronchoscopy Guidelines Committee, a subcommittee of the Standards of Care Committee of the British
Thoracic Society. British Thoracic Society guidelines on diagnostic flexible bronchoscopy. Thorax 2001; 56: Suppl. 1, i1-i21.

2 Gonzalez R, De-La-Rosa-Ramirez I, Maldonado-Hernandez A, et al. Should patients undergoing a bronchoscopy be sedated? Acta Anaesthesiol Scand 2003; 47: 411-415.

3 Putinati S, Ballerin L, Corbetta L, et al. Patient satisfaction with conscious sedation for bronchoscopy. Chest 1999; 115: 1437-1440.

4 Matot I, Kramer MR. Sedation in outpatient bronchoscopy. Respir Med 2000; 94: 1145-1153.

5 Bernasconi M, Chhajed PN, Muller P, et al. Patients' satisfaction with flexible bronchoscopy in a hospital-based community practice. Respiration 2009; 78: 440-445.

6 Pickles J, Jeffrey M, Datta A, et al. Is preparation for bronchoscopy optimal? Eur Respir J 2003; 22: 203-206.

7 Chhajed PN, Wallner J, Stolz D, et al. Sedative drug requirements during flexible bronchoscopy. Respiration 2005; 72: 617-621.

8 Stolz D, Chhajed PN, Leuppi J, et al. Nebulized lidocaine for flexible bronchoscopy: a randomized, double-blind, placebocontrolled trial. Chest 2005; 128: 1756-1760.

9 Stolz D, Pollak V, Chhajed PN, et al. A randomized, placebocontrolled trial of bronchodilators for bronchoscopy in patients with COPD. Chest 2007; 131: 765-772.

10 Stolz D, Chhajed PN, Leuppi JD, et al. Cough suppression during flexible bronchoscopy using combined sedation with midazolam and hydrocodone: a randomised, double blind, placebo controlled trial. Thorax 2004; 59: 773-776.

11 Stolz D, Kurer G, Meyer A, et al. Propofol versus combined sedation in flexible bronchoscopy: a randomised non-inferiority trial. Eur Respir J 2009; 34: 1024-1030.

12 Fulton B, Sorkin EM. Propofol. An overview of its pharmacology and a review of its clinical efficacy in intensive care sedation. Drugs 1995; 50: 636-657.

13 Shelley MP, Wilson P, Norman J. Sedation for fibreoptic bronchoscopy. Thorax 1989; 44: 769-775.

14 Clark G, Licker M, Younossian AB, et al. Titrated sedation with propofol or midazolam for flexible bronchoscopy: a randomised trial. Eur Respir J 2009; 34: 1277-1283.

15 Bryson HM, Fulton BR, Faulds D. Propofol. An update of its use in anaesthesia and conscious sedation. Drugs 1995; 50: 513-559.

16 Hara M, Kai Y, Ikemoto Y. Propofol activates GABAA receptorchloride ionophore complex in dissociated hippocampal pyramidal neurons of the rat. Anesthesiology 1993; 79: 781-788.

17 Zed PJ, Abu-Laban RB, Chan WW, et al. Efficacy, safety and patient satisfaction of propofol for procedural sedation and analgesia in the emergency department: a prospective study. CJEM 2007; 9: 421-427.

18 Berkenbosch JW, Graff GR, Stark JM, et al. Use of a remifentanilpropofol mixture for pediatric flexible fiberoptic bronchoscopy sedation. Paediatr anaesthe 2004; 14: 941-946.

19 Hohl CM, Nosyk B, Sadatsafavi M, et al. A cost-effectiveness analysis of propofol versus midazolam for procedural sedation in the emergency department. Acad Emerg Med 2008; 15: 32-39.

20 Rodrigo MR, Jonsson E. Conscious sedation with propofol. Br Dent J 1989; 166: 75-80.

21 Gasparovic S, Rustemovic N, Opacic M, et al. Clinical analysis of propofol deep sedation for 1,104 patients undergoing gastrointestinal endoscopic procedures: a three year prospective study. World J Gastroenterol 2006; 12: 327-330.

22 Clarkson K, Power CK, O'Connell F, et al. A comparative evaluation of propofol and midazolam as sedative agents in fiberoptic bronchoscopy. Chest 1993; 104: 1029-1031.

23 Chhajed PN, Glanville AR. Management of hypoxemia during flexible bronchoscopy. Clin Chest Med 2003; 24: 511-516.

24 Heuss LT, Schnieper P, Drewe J, et al. Safety of propofol for conscious sedation during endoscopic procedures in high-risk patients - a prospective, controlled study. Am J Gastroenterol 2003; 98: 1751-1757. 
25 Facciolongo N, Patelli M, Gasparini S, et al. Incidence of complications in bronchoscopy. Multicentre prospective study of 20,986 bronchoscopies. Monaldi Arch Chest Dis 2009; 71: 8-14.

26 Bosslet GT, Devito ML, Lahm T, et al. Nurse-administered propofol sedation: feasibility and safety in bronchoscopy. Respiration 2010; 79: 315-321.
27 Dang D, Robinson P, Winnicki S, et al. The safety of flexible fiberoptic bronchoscopy and proceduralist-administered sedation: a tertiary referral centre experience. Intern Med J 2010; [Epub ahead of print DOI: 10.1111/j.1445-5994.2010.02261.x].

28 Dinis-Ribeiro M, Vargo JJ. Sedation by non-anesthesiologists: are opioids and benzodiazepines outdated? Digestion 2010; 82: 100-101. 